\title{
Hybrid Flow-Shop Scheduling (HFS) Problem Solving with Migrating Birds Optimization (MBO) Algorithm
}

\author{
Yona Eka Pratiwi, Kusbudiono*, Abduh Riski, Alfian Futuhul Hadi \\ Department of Mathematics, The University of Jember, Indonesia
}

Received July 21, 2019; Revised September 25, 2019; Accepted February 20, 2020

Copyright $\mathrm{C} 2020$ by authors, all rights reserved. Authors agree that this article remains permanently open access under the terms of the Creative Commons Attribution License 4.0 International License

\begin{abstract}
The development of an increasingly rapid industrial development resulted in increasingly intense competition between industries. Companies are required to maximize performance in various fields, especially by meeting customer demand with agreed timeliness. Scheduling is the allocation of resources to the time to produce a collection of jobs. PT. Bella Agung Citra Mandiri is a manufacturing company engaged in making spring beds. The work stations in the company consist of 5 stages consisting of ram per with three machines, clamps per 1 machine, firing mattresses with two machines, sewing mattresses three machines and packing with one machine. The model problem that was solved in this study was Hybrid Flowshop Scheduling. The optimization method for solving problems is to use the metaheuristic method Migrating Birds Optimization. To avoid problems faced by the company, scheduling is needed to minimize makespan by paying attention to the number of parallel machines. The results of this study are scheduling for 16 jobs and 46 jobs. Decreasing makespan value for 16 jobs minimizes the time for 26 minutes 39 seconds, while for 46 jobs can minimize the time for 3 hours 31 minutes 39 seconds.
\end{abstract}

Keywords MBO, HFS, Metaheuristic, Deviation Percentage Algorithm, Makespan

\section{Introduction}

Mathematics is one part of science that plays an important role in the world of technology and companies. The industry that is growing rapidly along with the increasingly modern technological sophistication causes the level of competition between industries increasingly tight, and companies must maximize performance in various fields. One of them is to fulfill customer demand with the agreed timelines. This aspect is related to the production scheduling system.

Scheduling is a process of allocating resources, especially machines that are limited to completing several different jobs. Scheduling functions to optimize the time of completion of a job with a limited machine so that it can meet the production target so that the company can determine the right scheduling system and following the competitive situation and strategy implemented. One of the production scheduling systems in industry is the flow shop scheduling system.

The simplest flow shop scheduling is flow shop scheduling on a single production line, which is only one machine for each stage of its operation. While the problem that often arises in some companies is flow shop scheduling on parallel production lines, namely hybrid flow shop scheduling with one or more services that have more than one machine, hybrid flow shop scheduling is one of the schedules of sophisticated flow shop which consists of various stages (stages) of production processes and materials to be processed in the same direction. One of the essential goals in scheduling is to increase the efficiency and effectiveness of production by minimizing makespan to meet consumer demand on time, and therefore, a solution is needed by creating an effective scheduling system.

In developing mathematics, the optimization method for completing hybrid flow shop scheduling is growing. In the search for efficient and comprehensive solutions, the metaheuristic method uses mechanisms that mimic social behavior or strategies that exist in nature. Although there is no guarantee that the answer found is the optimal solution, a well-built metaheuristic method can provide a solution that approaches the optimal solution. [1]

In the HFS problem, one of the optimization methods is the Migrating Birds Optimization (MBO) algorithm. It is a metaheuristic algorithm that is capable of solving QAP (Quadratic Assignment Problem) problems [2]. So we are interested in implementing the Migrating Birds Optimization algorithm on other issues, namely Hybrid 
Flow Shop Scheduling to represent the effectiveness and efficiency of the Migrating Birds Optimization algorithm based on the smallest makespan aspect.

Based on the description above, the exciting thing that we will examine is how to implement the Migrating Birds Optimization algorithm in Hybrid flow shop scheduling and the solutions provided by it. Next, we will compare the results of the solution, to apply and know the solutions provided by the Migrating Birds Optimization algorithm in Hybrid flow shop scheduling. So that the benefits of this writing are to obtain an optimal solution, namely the results of schedule with a minimum makespan value, and can provide information about the Migrating Birds Optimization algorithm and Hybrid flow shop scheduling for readers.

Scheduling is a work order arrangement plan and allocation of resources, both in the form of time and facilities for each process of completing an operation. Scheduling is the first step to planning the entire production process because scheduling is a very important activity in the production process. Scheduling problems will arise when at a certain stage, several jobs must be completed at the same time, but the number of machines or other production facilities is limited. The effort to overcome this problem is to schedule some of the work so that later we will get the most optimal work order.

In the production process, there are three elements of scheduling, namely job, operation, and machine. Job is a job. By completing the work, we will get a product. Operations are part of the process of a job, to complete a job requires a work operation; Machines are the resources needed to complete the job completion process. With the existence of these elements, the purpose of scheduling is to increase the productivity of the machine, reduce the inventory of semi-finished goods, reduce work delays and reduce the total time required

Flow shop scheduling is the production process scheduling of each $\mathrm{n}$ job, which has a sequence of production processes and through the same m machine [3]. The machine penetrates each job in the same order at least on one machine, and one machine can process at most one job. The description of the flow shop scheduling model is a set of $\mathrm{M}$ machines, $\mathrm{M}=\{1,2, \ldots, m\}$ which is used to process a number of $\mathrm{N}$ jobs, $\mathrm{N}=\{1,2, \ldots, n\}$ [4]. Each machine can only process one job at the same time and once at each stage.

Hybrid flow shop scheduling is the development of flow shop scheduling problems that have parallel machines at each stage. Hybrid flow shop is a system consisting of various stages (stages) of production and material processes. The Hybrid flow shop processes these stages in a directional flow, where there is at least one stage in the production process that has identical machines and is arranged in parallel [5]. In the HF scheduling system, each stage can consist of 1 or more identical machines [6].

The use of Lower Bound (LB) can accommodate the absence of information about the value of the optimal solution. Makespan is a standard criterion for HFS problems. The Lower Bound value can be calculated using equation (1)

$$
\begin{gathered}
L B=\max _{i=1, \ldots, k}\left\{\min _{j \in J}\left\{\sum_{l=1}^{i-1} t_{l j}\right\}+\max \left\{M_{1}(i), M_{2}(i\right.\right. \\
)\}+\min _{j \in J}\left\{\sum_{l=i+1}^{k} t_{l j}\right\}\right\}
\end{gathered}
$$

where,

$$
\begin{gathered}
M_{1}(i)=\left\lceil\frac{1}{m_{i}} \sum_{j \in J} t_{i j} \cdot \text { size }_{i j}\right\rceil, \\
M_{2}=\sum_{j \in A_{i}} t_{i j}+\left\lceil\frac{1}{m_{i}} \sum_{j \in B_{i}} t_{i j} \cdot \text { size }_{i j}\right], \\
A_{i}=\left\{j \mid \text { size }_{i j}>\frac{m_{i}}{2}\right\}, B_{i}=\left\{j \mid \text { size }_{i j}=\frac{m_{i}}{2}\right\} .
\end{gathered}
$$

To see the comparison between makespan and Lower Bound, we do this by calculating the percentage deviation or the Deviation Percentage Algorithm (PDA). Calculation of percentage of deviations is as follows:

$$
P D_{A}(l)=100 \% \times \frac{C_{\max }(l)-L B}{L B}
$$

The algorithm Migrating Birds Optimization (MBO) is a metaheuristic algorithm inspired by nature based on a "V" shaped the formation of migratory birds, which has proven to be an effective formation in energy savings [2].

Solving optimization problems using the MBO algorithm [7] is as follows:

1. Initialization of the Initial Population, the initial positioning of the population in the MBO algorithm, is random, which is the solution in the search space for optimal value and has a permutation solution.

2. Environment and Solution Sharing, flocks of birds on the right or left side of the lead bird represent a neighboring solution. Determination or analysis of results from neighboring solutions is carried out iterations produce maximum values. To improve the current solution, we use the best neighbor solution that has been obtained by the previous process. The number of neighboring solutions made and the number of shared neighbor solutions are given as parameters, and this parameter is determined as follows:

$$
\begin{gathered}
k \in\{3,5,7, \ldots\} \\
x \in\left\{1,2,3, \ldots, \frac{(k-1)}{2}\right\} \\
n=k-x
\end{gathered}
$$

Information:

$\mathrm{n}=$ number of neighbor solutions except sharing

$\mathrm{k}=$ number of neighboring solutions that we must consider

$\mathrm{x}=$ number of neighbor solutions for us to share with the 
next solution.

The generation of a neighboring solution for Hybrid Flows problems we help with the SWAP method or what is often known as swapping permutation. We do the swapping by generating random numbers $i$ and $j$. This random number shows position $i$ and position $j$. This swapping process works by exchanging jobs in position $i$ with jobs in position $j$.

3. Termination Criteria, the iteration process will stop when it reaches the stop criteria. Criteria for dismissal of the Migrating Birds Optimization (MBO) algorithm is the iteration that has been carried out has reached the maximum iteration

\section{Materials and Methods}

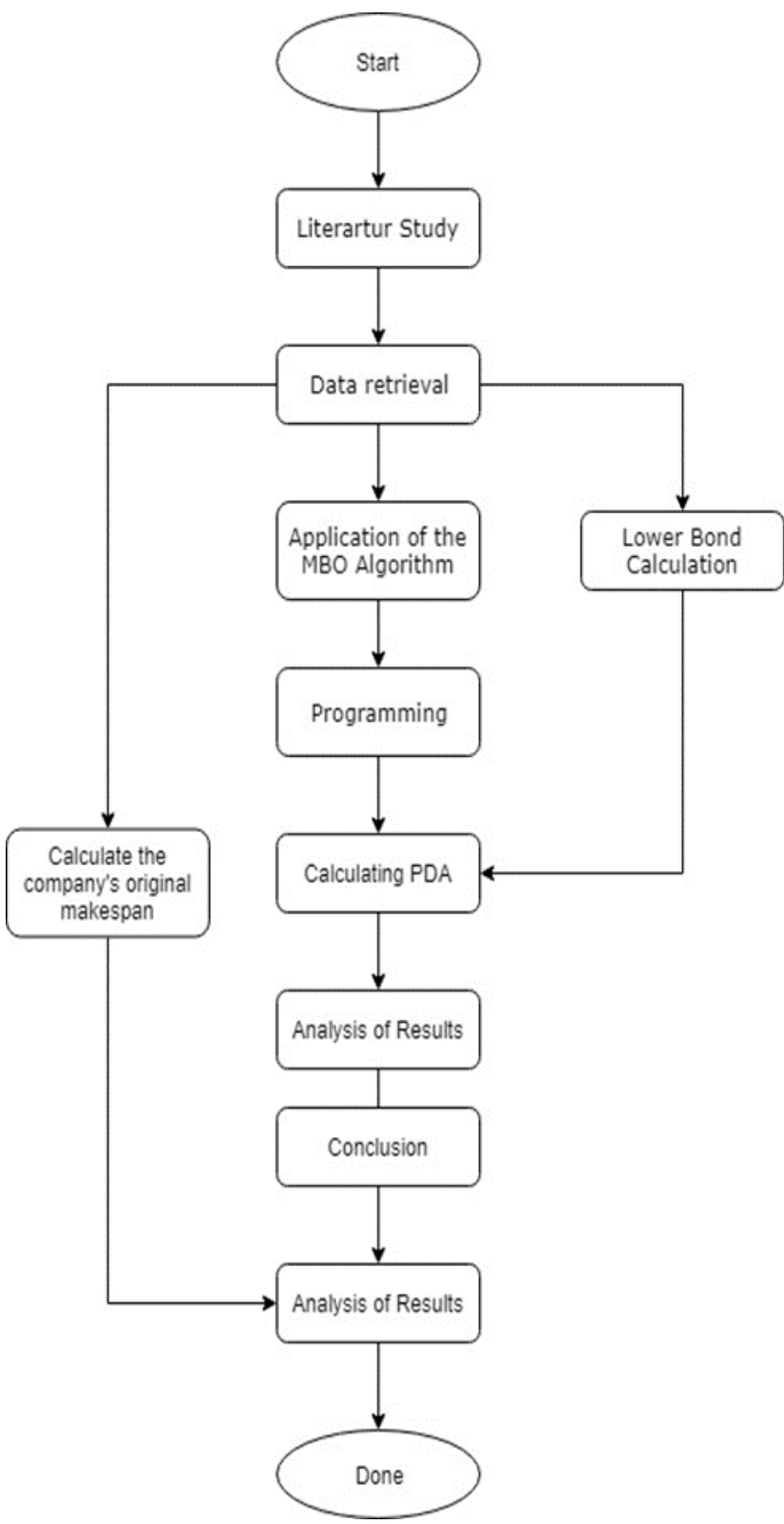

Figure 1. Flowchart research steps
The data used in this research is secondary data taken from PT Bella Agung Citra Mandiri. The research steps used are collecting literature related to the MBO algorithm to know and understand the MBO algorithm. Then collect the data needed for research from the company. We know that the company has five stages of each size and type of mattress, and also with several different machines. Next, we will apply the MBO algorithm to scheduling Hybrid Flow shop. After that, the making of programs and program simulations using two types of data, namely 16 jobs data and 46 jobs. Followed by analyzing the results of the program, then comparing the makespan of the program with the company's original makespan to find out the value of the PDA so that we can conclude. We can see the flowchart of the research steps in Figure 1.

In this study, we conducted experiments according to the data taken by comparing the makespan that we applied to two data. It is data 5 stages 16 jobs and data 5 stages 46 jobs in a maximum of different iterations. The parameter values used are population parameters and their equivalence with a combination of 5,15 , and 25 in each experiment. For sharing solutions, we make varied. We test each parameter value with a maximum iteration of 500 and 1000. From each combination we make, the program runs ten times.

The results we obtained from the MBO algorithm on Hybrid flow shop scheduling problems indicate that parameter $x$ greatly influences the achievement of optimal solutions. When the sharing parameter is worth 1 , the chances of the solution we get are getting closer to optimal, and the converging is relatively faster, but the computation time is relatively longer. It is because the smaller the value of the sharing parameter, the neighboring solution except the sharing that we generate is more and more. So that candidates for new solutions are also more than before. Thus the possibility of getting an optimal solution is higher. Because of the neighboring solution except the sharing that we generate is more and more, the calculation process except sharing is also increasing so that the computation time is getting longer.

In this Hybrid flow shop scheduling problem, the $\mathrm{MBO}$ algorithm helps solve problems that exist in a company. Based on the testing of small data and large company data, convergence and running time are affected by the parameters used. In general, the use of parameters in the MBO algorithm is relatively influential on the results of the makespan in each job. But if we look at the average results obtained, to get the optimal solution, the population used is very influential on the results of convergence and running time. We get the optimal solution quickly if the population that generates $(\mathrm{m})$ in large numbers and consider the neighbor solution $(\mathrm{k})$ is a relatively large neighbor solution and the smallest number of sharing.

After obtaining the most optimal parameter values, then do the final simulation to complete all data (16 jobs and 46 jobs) with these parameter values. The parameters are 
population number $(\mathrm{m})=75$, number of neighboring solutions $(\mathrm{k})=25$, sharing $(\mathrm{x})=1$ with a maximum iteration of 1000 . We can see the results of applying the Hybrid Flow shop scheduling solution using data from PT Bella Agung Citra Mandiri in Table 1 and Table 2.

With the data of 16 company jobs for mattress production, we get makespan 15655 which requires a production time of 5 hours 21 minutes 5 seconds with a job sequence 1-2-3-4-5-6-7-8-9-10-11-12 -13-14-15-16, while the optimal makespan result from the program is 14066 . Therefore, we can see that the work on Hybrid Flowshop scheduling with the Migrating Birds Optimization algorithm can minimize the time for 26 minutes 29 seconds.

Table 1. Final Simulation Data is 5 stage 16 jobs with 1000 Iterations

\begin{tabular}{|c|c|c|c|c|}
\hline Experiment & Makespan & Convergent Iteration & Computation Time & $\operatorname{PDA}(\%)$ \\
\hline 1 & 14066 & 5 & 606.6423 & $21.3109 \%$ \\
\hline 2 & 14066 & 5 & 608.0631 & $21.3109 \%$ \\
\hline 3 & 14066 & 11 & 620.0266 & $21.3109 \%$ \\
\hline 4 & 14066 & 6 & 611.3195 & $21.3109 \%$ \\
\hline 5 & 14066 & 6 & 612.4782 & $21.3109 \%$ \\
\hline 6 & 14066 & 7 & 611.3553 & $21.3109 \%$ \\
\hline 7 & 14066 & 13 & 613.6233 & $21.3109 \%$ \\
\hline 8 & 14066 & 4 & 612.9036 & $21.3109 \%$ \\
\hline 9 & 14066 & 9 & 611.6245 & $21.3109 \%$ \\
\hline 10 & 14066 & 10 & 614.0748 & $21.3109 \%$ \\
\hline
\end{tabular}

Table 2. Final Simulation Data is 5 stage 46 jobs with 1000 Iterations

\begin{tabular}{|c|c|c|c|c|}
\hline Experiment & Makespan & Convergent Iteration & Computation Time & PD $_{\mathrm{A}}(\%)$ \\
\hline 1 & 35430 & 209 & 1726.3200 & $3.2042 \%$ \\
\hline 2 & 35529 & 133 & 1743.2942 & $3.4926 \%$ \\
\hline 3 & 35302 & 148 & 1724.8602 & $2.8313 \%$ \\
\hline 4 & 35430 & 209 & 1799.0247 & $3.2042 \%$ \\
\hline 5 & 35482 & 136 & 1718.3556 & $3.3557 \%$ \\
\hline 6 & 35330 & 158 & 1767.2683 & $2.9129 \%$ \\
\hline 7 & 35297 & 165 & 1740.2812 & $2.8168 \%$ \\
\hline 8 & 35430 & 209 & 1794.7720 & $3.2042 \%$ \\
\hline 9 & 35529 & 133 & 1730.5848 & $3.4926 \%$ \\
\hline 10 & 35313 & 249 & 1729.5146 & $2.8634 \%$ \\
\hline
\end{tabular}

Table 3. Final Simulation Data is 5 stage 46 jobs with 500 Iterations

\begin{tabular}{|c|c|c|c|c|}
\hline Experiment & Makespan & Convergent Iteration & Computation Time & PDA (\%) \\
\hline 1 & 35395 & 25 & 4459.7560 & $3,1022 \%$ \\
\hline 2 & 35278 & 82 & 4378.4267 & $2,7614 \%$ \\
\hline 3 & 35299 & 105 & 4297.5180 & $2,8226 \%$ \\
\hline 4 & 35258 & 132 & 4517.6693 & $2,7032 \%$ \\
\hline 5 & 35302 & 182 & 4472.4839 & $2,8313 \%$ \\
\hline 6 & 35419 & 82 & 4352.4709 & $3,1722 \%$ \\
\hline 7 & 35507 & 79 & 4527.8675 & $3,4285 \%$ \\
\hline 8 & 35506 & 119 & 4328.3257 & $3.4560 \%$ \\
\hline 9 & 35258 & 132 & 4545.4647 & $2.7032 \%$ \\
\hline 10 & 35365 & 63 & 4383.7481 & $3.0149 \%$ \\
\hline
\end{tabular}


From the results of the final simulation above for the 5 stage, 16 job data we say optimal because of the ten times running, we obtained the same makespan value. Whereas for the five stages $46 \mathrm{job}$ data, there is still the possibility to find the optimal solution. To find the best makespan of the data, the step we need to do is to enlarge the population parameters and neighboring solutions. The parameters we use are the population $(\mathrm{m})=125$, the number of neighboring solutions $(\mathrm{k})=75$, sharing $(\mathrm{x})=1$ with an iteration of 500. We can see the results of the study in Table 3.

On the HF scheduling problem for data on five stages 46 jobs with the MBO algorithm, we get the optimal solution for the best makespan, which is 35258 with converging iterations at 132. The computing time is 4517.6693 seconds, and the PDA is $2.7032 \%$. From the results of makespan, the production time of the company's mattress is 1 day 1 hour 47 minutes 38 seconds with the order of jobs 10-20-46-45-19-40-44-37-39-41-35-18-27-43

$-5-23-14-2-36-38$

-31-21-29-13-34-42-3-26-16-22-9-8-1-30-11-24-28-33-4

$-12-6-7-32-25-15-17$.

\section{Conclusions}

Based on the results and our discussion, we conclude the following:

1. The Migrating Birds Optimization algorithm can help us solve HF Scheduling problems. We obtain makespan by entering time, size and engine data on several stages, with several parameters combined including population $(\mathrm{m})=5,15,25$; neighboring solutions $(\mathrm{k})=5,15,25$; sharing value $(\mathrm{x})=1,2,4,7$, 8,12 ; and lots of iterations $=500,1000$.

2. The Migrating Birds Optimization algorithm is effective, because it produces a makespan value that approaches the optimal value based on Average Percentage Deviation. In the data of 16 job 5 stage the best makespan is 14066 seconds, and for data 46 jobs 5 stages is 35258 seconds. While the parameters for data 16 jobs 5 stages are population $(\mathrm{m})=75$; neighboring solutions $(\mathrm{k})=25$; sharing value $(\mathrm{x})=1$; $\operatorname{imax}=1000$; and for data 16 jobs 5 stages in the population parameter $(\mathrm{m})=125$; neighboring solution $(\mathrm{k})=75$; sharing value $(\mathrm{x})=1 ; \operatorname{imax}=500$.

\section{Acknowledgements}

The University of Jember supported this research. For the preparation of this paper, we thanks to all member of Pemodelan Matematika Research Group and all member of the Mathematics Mod-elling and Computation Laboratory, Department of Mathematics of UNEJ.

\section{REFERENCES}

[1] F. S. Hillier, G. J. Lieberman GJ. Introduction to Operations Research: Ninth edition, Standford University, 2010.

[2] E. Duman, M. Uysal, A. F. Alkaya. Migrating Birds Optimization: a new metaheuristic approach and its performance on quadratic assignment problem, Information Science, Vol.217, 65 - 77, 2012.

[3] K. Baker. Introduction to Sequencing and Scheduling, John Wiley and Sons Inc, New York, 1997.

[4] S.R. Hejazi, S. Saghafian. Flowshop-Scheduling Problems with Makespan Criterion: A Review, International Journal of Production Research, Vol.43, No.14, 2895-2929, 2005.

[5] T. Uetake, H. Tsubone, M. Ohba M. A Production Scheduling System in A Hybrid Flow Shop, International Journal of Production Ecomonics, Vol.41, 395-398, 1995.

[6] F. S. Serifoglu, G. Ulusoy. Mulitiprocessor Task Scheduling in Multistage Hybrid Flow Shop: A Genetic Algorithm Approach, Journal of Operational Research Society, Vol.55, No.5, 504-512, 2004.

[7] E. Ulker, V. Tongur. Migrating birds optimization (MBO) algorithm to solve knapsack problem, Procedia Computer Science, Vol.111, 71 - 76, 2017. 